PUPT-1842

hep-th/9903128

\title{
Intersecting D-branes and black holes in type 0 string theory
}

\author{
Miguel S. Costa円 \\ Joseph Henry Laboratories \\ Princeton University \\ Princeton, New Jersey 08544, USA
}

\begin{abstract}
We study intersecting D-branes in type 0 string theories and show that the $\mathrm{D} p_{ \pm^{-}}$ brane bound states obey similar intersecting rules as the $\mathrm{D} p$-branes of the type II theories. The $\mathrm{D} 5_{ \pm}-\mathrm{D} 1_{ \pm}$brane system is studied in detail. We show that the corresponding near-horizon geometry is the $A d S_{3} \times S^{3} \times T^{4}$ space and that there is no tachyon instability in this background. The Bekenstein-Hawking entropy is calculated. The worldvolume theory on the $\mathrm{D} 5_{ \pm}-\mathrm{D} 1_{ \pm}$system is also studied. This theory contains both bosons and fermions and it is seen to arise as a projection of the supersymmetric gauge theory related to the D5-D1 system of the type IIB theory. The Bekenstein-Hawking entropy formula is reproduced exactly using the dual conformal field theory.
\end{abstract}

\footnotetext{
${ }^{1}$ miguel@feynman.princeton.edu
} 


\section{Introduction}

Recently there has been a renewed interest in the type 0 string theories [1, 2]. While in the type II theories the GSO projection acts independently on the left- and right-moving sectors, in the type 0 theories this projection ties both sectors together. More precisely, this so-called diagonal GSO projection is just $\left(1+(-1)^{F+\tilde{F}}\right) / 2$ in the NS - NS sectors and $\left(1 \pm(-1)^{F+\tilde{F}}\right) / 2$ in the $\mathrm{R}-\mathrm{R}$ sectors (the plus sign choice gives the type 0B theory and the minus sign the type 0A theory). The resulting string theories have worldsheet supersymmetry but no space-time supersymmetry. The closed string spectrum is divided in the following sectors (in the notation of [3])

$$
\begin{array}{ll}
0 A: & (\mathrm{NS}-, \mathrm{NS}-) \oplus(\mathrm{NS}+, \mathrm{NS}+) \oplus(\mathrm{R}+, \mathrm{R}-) \oplus(\mathrm{R}-, \mathrm{R}+), \\
0 \mathrm{~B}: & (\mathrm{NS}-, \mathrm{NS}-) \oplus(\mathrm{NS}+, \mathrm{NS}+) \oplus(\mathrm{R}+, \mathrm{R}+) \oplus(\mathrm{R}-, \mathrm{R}-) .
\end{array}
$$

Notice that these theories have no fermions in the bulk. The massless bosonic fields are similar to the type II theories with the $\mathrm{R}-\mathrm{R}$ fields doubled. In addition, there is a tachyon with $m^{2}=-2 / \alpha^{\prime}$ coming from the (NS-, NS-) sector.

The doubling of the $\mathrm{R}-\mathrm{R}$ sector in the type 0 theories has some interesting conse-

quences. For each $p$ (even or odd in the $0 \mathrm{~A}$ or $0 \mathrm{~B}$ theories, respectively) there are two $(p+1)$-form gauge potentials $\mathcal{A}_{p+1}$ and $\overline{\mathcal{A}}_{p+1}$ corresponding to the $\mathrm{R}-\mathrm{R}$ sectors in (1.1). Thus, these theories will have two different types of $\mathrm{D} p$-branes and corresponding antibranes [1]. It is convenient to redefine the gauge fields and corresponding D-brane charges in the following way $[4$

$$
\left(\mathcal{A}_{p+1}\right)_{ \pm}=\frac{1}{\sqrt{2}}\left(\mathcal{A}_{p+1} \pm \overline{\mathcal{A}}_{p+1}\right), \quad q_{ \pm}=\frac{1}{2}(q \pm \bar{q})
$$

In this notation we have $\mathrm{D} p_{+}$-branes, $\mathrm{D} p_{-}$-branes and the corresponding anti-branes. A $\mathrm{D} p_{+}$-brane carries the charges $q=1=\bar{q}$ and a $\mathrm{D} p_{-}$-brane the charges $q=1=-\bar{q}$. In the type $0 \mathrm{~B}$ theory and for $p=3$, the self-dual field strength $\mathcal{F}_{5}=d \mathcal{A}_{4}$ and the anti-self-dual field strength $\overline{\mathcal{F}}_{5}=d \overline{\mathcal{A}}_{4}$ may be combined to form an unconstrained 5-form field strength. Then the D3+-brane will be electrically charged and the D3_-brane magnetically charged.

Following a suggestion made by Polyakov [5], and the earlier work on the $A d S / C F T$ duality [6, 7, 8], Klebanov and Tseytlin [4] used the type 0 string theory to study nonsupersymmetric non-conformal gauge theories. In particular, they considered the theory of $N$ coincident electrically charged D3-branes (D3 + -branes). The corresponding effective worldvolume theory is purely bosonic and tachyon free. More precisely, it is the fourdimensional $U(N)$ gauge theory with six adjoint scalars. It was then argued that the $\mathrm{R}-\mathrm{R}$ flux in the type 0 dual background stabilizes the tachyon, as expected from the 
duality equivalence. Since then a few papers reporting results on this model appeared [9-14] (see also [15, 16, 17] for related work in non-critical strings).

In a related development, Klebanov and Tseytlin [18] also considered the theory of $N$ D3+-branes coincident with $N$ D3_-branes ( $N$ coincident D3 $3_{ \pm}$-branes 2 ). The novelty here is that the worldvolume theory for this bound state has fermions corresponding to open strings with one end on a D3+-brane and the other end on a D3_-brane. This theory is the conformal $U(N) \times U(N)$ gauge theory with six adjoint scalars and eight bifundamental Weyl spinors (four in the $(N, \bar{N})$ plus four in the $(\bar{N}, N)$ ). The dual type 0 background is the $A d S_{5} \times S^{5}$ space. Remarkably, the $\mathrm{D} p_{ \pm}$-brane bound states of the type 0 theories share many properties of the BPS D -branes of the type II theories. In particular, two of such parallel branes satisfy the no-force condition [4, 18] (see also [12, 19] for details on the potentials between different type 0 D3-branes).

The results described above lead us to consider the problem of intersecting $\mathrm{D} p$-branes in the type 0 theories. We shall start in section two by studying the forces between intersecting type 0 D-branes. We conclude that the $\mathrm{D} p_{ \pm}$-brane bound states may be intersected according to the same rules of the type II theories. In section three we consider the $D 5_{ \pm}-D 1_{ \pm}$brane system and show that the corresponding near-horizon geometry is the $A d S_{3} \times S^{3} \times T^{4}$ space. In this background the tachyon field is stabilized. We calculate the Bekenstein-Hawking entropy in terms of the quantized D-brane charges. In section four we study the worldvolume theory associated to the $\mathrm{D} 5_{ \pm}-\mathrm{D} 1_{ \pm}$brane system. This theory contains fermions corresponding to strings with one end on a $\mathrm{D} p_{+}$-brane and the other end on a $\mathrm{D} p_{-}^{\prime}$-brane. Also, we show that this gauge theory is a $Z_{2}$ projection of the type II gauge theory for the D5-D1 brane system, i.e. the compactification to two dimensions of a $N=1, D=6$ supersymmetric gauge theory [20]. By choosing an appropriate branch of our gauge theory, we define the dual conformal field theory and reproduce exactly the Bekenstein-Hawking entropy formula. We present our conclusions in section five.

\section{Intersecting D-branes in type 0 string theory}

We shall start by reviewing the results presented in [⿴囗十 for the interacting energy between parallel D-branes. The type 0 D-branes were earlier studied in [21] by using the boundary state formalism (see also [22, 23, 24] for related work on the open string descendents of the type 0B theory). The cylinder amplitude between two likely charged parallel $\mathrm{D} p$-branes

\footnotetext{
${ }^{2} \mathrm{~A} \mathrm{D} p_{ \pm}$-brane is a bound state of a $\mathrm{D} p_{+}$-brane and a $\mathrm{D} p_{-}$-brane; a $\mathrm{D} p_{\mp}$-brane is a bound state of a $\mathrm{D} p_{+}$-brane and a anti $\mathrm{D} p_{-}$-brane; similar definitions follow for the anti $\mathrm{D} p_{ \pm^{-}}$and $\mathrm{D} p_{\mp^{-}}$-branes.
} 
(i.e. between two $\mathrm{D} p_{+}$-branes or two $\mathrm{D} p_{-}$-branes) is obtained by omitting the $\mathrm{R}$ sector in the open string channel [21, 4]. The result is

$$
A=V_{p+1} \int_{0}^{\infty} \frac{d t}{2 t}\left(8 \pi^{2} \alpha^{\prime} t\right)^{-\frac{p+1}{2}} e^{-\frac{t Y^{2}}{2 \pi \alpha^{\prime}}}\left(\left[\frac{f_{3}(q)}{f_{1}(q)}\right]^{8}-\left[\frac{f_{4}(q)}{f_{1}(q)}\right]^{8}\right),
$$

where $q=e^{-\pi t}$. The first term in the parenthesis comes from the NS spin structure and the second term from the $\mathrm{NS}(-1)^{F}$ spin structure. The open string tachyon is projected out and the open string states are purely bosonic. In the closed string channel the amplitude (2.1) is seen to contain a term from the closed string tachyon, a term from the attractive NS - NS massless exchange and a term from the repulsive $\mathrm{R}-\mathrm{R}$ massless exchange (which is twice the NS - NS attractive term because the $\mathrm{R}-\mathrm{R}$ fields are doubled).

To calculate the cylinder amplitude between a $\mathrm{D} p_{+}$-brane and a $\mathrm{D} p_{-}$-brane, the correct projection in the open string channel is to retain the $\mathrm{R}$ sector only. The result is minus the amplitude in (2.1). However, in this case the open strings are associated with fermionic states. In the closed string channel we have a term from the tachyon and an attractive term from the NS - NS massless exchange (the $\mathrm{R}-\mathrm{R}$ contribution to the interacting potential cancels).

A few interesting results follow from the previous analysis [4]. Firstly, the worldvolume theory of a $\mathrm{D} p_{ \pm}$-brane bound state contains fermions arising from strings ending on branes with different charges and bosons arising from strings ending on likely charged branes. Secondly, the cylinder amplitude between two $\mathrm{D} p_{ \pm}$-branes may be seen to vanish by adding the contributions from each constituent. Also, because $q=0$ for the $\mathrm{D} p_{ \pm}$-brane, it decouples from the bulk tachyon, i.e. it is not a source for such field (or $\bar{q}=0$ for the $\mathrm{D} p_{\mp}$-brane).

Now we turn to the case of intersecting D-branes. We want to calculate the interacting energy between a $\mathrm{D} p$-brane and a $\mathrm{D} p^{\prime}$-brane in the type 0 theories. This problem is analogous to the type II case [25]. The open strings stretching between these branes will have either $\mathrm{DD}, \mathrm{NN}$ or $\mathrm{ND}(\equiv \mathrm{DN})$ boundary conditions. The only T-duality invariant quantity is the number of directions along which the open strings satisfy ND boundary conditions because the DD and NN boundary conditions are related by T-duality. Taking into account that the ghost contribution will cancel two of the (DD or NN) coordinates we have $\sharp \mathrm{ND}=8-\sharp(\mathrm{NN}+\mathrm{DD})$. If $\sharp \mathrm{ND} \neq 0$ there are fermionic zero modes in the $\mathrm{NS}(-1)^{F}$ sector and therefore this sector will not contribute (as well as the $\mathrm{R}(-1)^{F}$ sector).

To calculate the cylinder amplitude between $+\left(\right.$ or - ) charged $\mathrm{D} p$ - and $\mathrm{D} p^{\prime}$-branes we have to omit the $\mathrm{R}$ sector in the open string channel. The result is

$$
A=V_{l+1} \int_{0}^{\infty} \frac{d t}{2 t}\left(8 \pi^{2} \alpha^{\prime} t\right)^{-\frac{l+1}{2}} e^{-\frac{t Y^{2}}{2 \pi \alpha^{\prime}}}\left[\frac{f_{3}(q)}{f_{1}(q)}\right]^{\sharp(\mathrm{NN}+\mathrm{DD})}\left[\frac{f_{2}(q)}{f_{4}(q)}\right]^{\sharp \mathrm{ND}},
$$


where the branes intersect over a $l$-brane. If $\sharp N D=0$ (and therefore $\sharp(N N+D D)=8$ ) we obtain the contribution from the NS spin structure in the amplitude (2.1) (to which the contribution from the $\mathrm{NS}(-1)^{F}$ spin structure must be added). To factorize the cylinder in the closed string channel we need to consider the limit $t \rightarrow 0$. The result is

$$
\left[\frac{f_{3}(q)}{f_{1}(q)}\right]^{\sharp(\mathrm{NN}+\mathrm{DD})}\left[\frac{f_{2}(q)}{f_{4}(q)}\right]^{\sharp \mathrm{ND}} \sim \frac{t^{\sharp(\mathrm{NN}+\mathrm{DD}) / 2}}{2^{\sharp N D} / 2}\left(e^{\pi / t}+(8-2 \sharp \mathrm{ND})+\mathcal{O}\left(e^{-\pi / t}\right)\right) .
$$

The first term comes from the closed string tachyon and the second term from the NS - NS massless exchange. Note that the absence of the $\mathrm{NS}(-1)^{F}$ sector reflects the fact that different $\mathrm{D}$-branes do not interact through the $\mathrm{R}-\mathrm{R}$ gauge fields 25]. Note also that the NS - NS massless exchange vanishes for $\sharp N D=4$ but the tachyonic term remains.

The cylinder amplitude between a $\mathrm{D} p_{+^{-}}$and a $\mathrm{D} p_{-}^{\prime}$-brane is obtained by retaining the open string $\mathrm{R}$ sector only. The result is

$$
A=-V_{l+1} \int_{0}^{\infty} \frac{d t}{2 t}\left(8 \pi^{2} \alpha^{\prime} t\right)^{-\frac{l+1}{2}} e^{-\frac{t Y^{2}}{2 \pi \alpha^{\prime}}}\left[\frac{f_{2}(q)}{f_{1}(q)}\right]^{\sharp(\mathrm{NN}+\mathrm{DD})}\left[\frac{f_{3}(q)}{f_{4}(q)}\right]^{\sharp \mathrm{ND}} .
$$

Thus, the worldvolume theory of a $\mathrm{D} p_{+^{-}}$and a $\mathrm{D} p_{-}^{\prime}$-brane will contain fermions on their intersection. For small $t$, we found

$$
-\left[\frac{f_{2}(q)}{f_{1}(q)}\right]^{\sharp(\mathrm{NN}+\mathrm{DD})}\left[\frac{f_{3}(q)}{f_{4}(q)}\right]^{\sharp \mathrm{ND}} \sim \frac{t^{\sharp(\mathrm{NN}+\mathrm{DD}) / 2}}{2^{\sharp \mathrm{ND} / 2}}\left(-e^{\pi / t}+(8-2 \sharp \mathrm{ND})+\mathcal{O}\left(e^{-\pi / t}\right)\right) .
$$

Comparing with (2.3) we see that the sign of the tachyonic term is reversed while the term corresponding to the NS - NS massless exchange is the same.

The potential between a $\mathrm{D} p_{ \pm}$- and a $\mathrm{D} p_{ \pm}^{\prime}$-brane can be calculated by adding the potentials between the constituents. The result is twice the sum of (2.2) and (2.4). In particular, for $\sharp N D=4$ there is no force between these bound states. This is analogous to the type II case where for $\sharp \mathrm{ND}=4$ we have a state preserving a fraction of the space-time supersymmetry, i.e. a stable BPS state. In the type II case we are led to study the D5-D1 brane system that corresponds to a 5-dimensional black hole in the dual gravitational description of the system [26, 27]. Here we shall study the $\mathrm{D} 5_{ \pm}-\mathrm{D} 1_{ \pm}$brane system of the type $0 \mathrm{~B}$ theory. The worldvolume theory of such a bound state will contain fermions associated to open strings with one end on a + charged D-brane and the other end on a - charged D-brane, and bosons associated to strings ending on + charged D-branes or on - charged D-branes.

Finally, we consider the case $\sharp N D=8$ which deserves a separate discussion? [25]. In

\footnotetext{
${ }^{3}$ Recently, and while this work was in progress, a paper considering the intersection of a D5 + -brane and a D5_-branes over a string appeared [28]. They used the existence of chiral fermions on the intersection to derive the anomalous Wess-Zumino coupling [29] for $\mathrm{D} p_{+_{-}}$and $\mathrm{D} p_{-}$-branes.
} 
this case there are no fermionic zero modes in the $\mathrm{R}(-1)^{F}$ sector which has to be included. To calculate the amplitude for $+($ or -$)$ charged $\mathrm{D} p$ - and $\mathrm{D} p^{\prime}$-branes we retain the NS open string sector. The result is

$$
A=V_{l+1} \int_{0}^{\infty} \frac{d t}{2 t}\left(8 \pi^{2} \alpha^{\prime} t\right)^{-\frac{l+1}{2}} e^{-\frac{t Y^{2}}{2 \pi \alpha^{\prime}}}\left[\frac{f_{2}(q)}{f_{4}(q)}\right]^{8} .
$$

For a $\mathrm{D} p_{+}-$and a $\mathrm{D} p_{-}^{\prime}$-brane we have the contributions from the $\mathrm{R}(-1)^{F}$ and $\mathrm{R}$ sectors with the result

$$
A=V_{l+1} \int_{0}^{\infty} \frac{d t}{2 t}\left(8 \pi^{2} \alpha^{\prime} t\right)^{-\frac{l+1}{2}} e^{-\frac{t Y^{2}}{2 \pi \alpha^{\prime}}}\left(1-\left[\frac{f_{3}(q)}{f_{4}(q)}\right]^{8}\right)
$$

As in the type II theories, the cylinder amplitude between a $\mathrm{D} p_{ \pm^{-}}$and a $\mathrm{D} p_{ \pm}^{\prime}$-brane such that $\sharp \mathrm{ND}=8$ will vanish by the abstruse identity.

\section{The $\mathrm{D} 5_{ \pm}-\mathrm{D} 1_{ \pm}$black hole solution}

In the remainder of this paper we shall study the $\mathrm{D} 5_{ \pm}-\mathrm{D} 1_{ \pm}$brane system. We start by considering the corresponding gravitational solution. The appropriate truncation of the type $0 \mathrm{~B}$ effective action is 4

$$
\begin{aligned}
S=\frac{1}{2 \kappa_{10}^{2}} \int d^{10} x \sqrt{-g}[ & e^{-2 \phi}\left(R+4\left(\partial_{a} \phi\right)^{2}-\frac{1}{4}\left(\partial_{a} T\right)^{2}-\frac{m^{2}}{4} T^{2}\right) \\
& \left.-\frac{1}{2.3 !} f_{+}(T)\left(\mathcal{F}_{3+}\right)^{2}-\frac{1}{2.3 !} f_{-}(T)\left(\mathcal{F}_{3-}\right)^{2}\right],
\end{aligned}
$$

where

$$
f_{ \pm}=1 \pm T+\mathcal{O}\left(T^{2}\right), \quad m^{2}=-2 / \alpha^{\prime}, \quad \kappa_{10}^{2}=(2 \pi)^{6} \pi g^{2} \alpha^{\prime 4}
$$

and $a$ is a ten-dimensional index. The dilaton field $\phi$ has its zero mode $\phi_{0}$ subtracted.

We proceed by compactifying the above action to five dimensions with the ansatz [30]

$$
\begin{aligned}
d s^{2} & =e^{\phi_{6}}\left[e^{-\frac{2}{3} \lambda} d s_{5}^{2}+e^{2 \lambda}\left(d x_{1}+\mathcal{A}_{\mu}^{(K)} d x^{\mu}\right)^{2}\right]+e^{2 \nu} d x^{I} d x_{I} \\
\phi & =\phi_{6}+2 \nu
\end{aligned}
$$

where $I, J, \ldots=6, \ldots, 9$ and $\mu, \nu, \ldots$ are five-dimensional indices. The field $\phi_{6}$ is the dilaton field in the six-dimensional theory. We shall make the following ansatz for the 3-form field strengths $\mathcal{F}_{3 \pm}$ and Kałuża-Klein field $\mathcal{A}_{\mu}^{(K)}:\left(\mathcal{F}_{3 \pm}\right)_{\mu \nu 1}$ are electric corresponding to 
the D1-branes, $\left(\mathcal{F}_{3 \pm}\right)_{\mu \nu \rho}$ are magnetic corresponding to the D5-branes and $\mathcal{A}_{\mu}^{(K)}$ is electric corresponding to the Kałuża-Klein modes along $x_{1}$. The action (3.1) becomes

$$
\begin{aligned}
S=\frac{1}{2 \kappa_{5}^{2}} \int & d^{5} x \sqrt{-g_{5}}\left[R-\left(\partial_{\mu} \phi_{6}\right)^{2}-\frac{4}{3}\left(\partial_{\mu} \lambda\right)^{2}-4\left(\partial_{\mu} \nu\right)^{2}-\frac{1}{4}\left(\partial_{\mu} T\right)^{2}\right. \\
& -\frac{m^{2}}{4} e^{\phi_{6}-\frac{2}{3} \lambda} T^{2}-\frac{1}{2.2 !} e^{-\frac{4}{3} \lambda+4 \nu}\left(f_{+}(T)\left(\mathcal{F}_{+\mu \nu 1}\right)^{2}+f_{-}(T)\left(\mathcal{F}_{-\mu \nu 1}\right)^{2}\right) \\
& \left.-\frac{1}{2.2 !} e^{\frac{8}{3} \lambda}\left(\mathcal{F}_{\mu \nu}^{(K)}\right)^{2}-\frac{1}{2.3 !} e^{\frac{4}{3} \lambda+4 \nu}\left(f_{+}(T)\left(\mathcal{F}_{+\mu \nu \rho}\right)^{2}+f_{-}(T)\left(\mathcal{F}_{-\mu \nu \rho}\right)^{2}\right)\right],
\end{aligned}
$$

where $\kappa_{5}^{2}=\kappa_{10}^{2} / V_{5}$ with $V_{5}$ the volume of the compact five-dimensional space $T^{5}$. Comparing with the results in [30 the new ingredients are the tachyon field and the doubling of the $\mathrm{R}-\mathrm{R}$ fields.

Next we perform the following ansatz for the fields in the reduced action (3.4)

$$
\begin{aligned}
& d s_{5}^{2}=-e^{2 a(r)} d t^{2}+e^{2 b(r)} d r^{2}+e^{2 c(r)} d \Omega_{3}^{2} \\
& \phi_{6}=\phi_{6}(r), \quad \lambda=\lambda(r), \quad \nu=\nu(r), \quad T=T(r), \\
& \mathcal{A}_{t}^{(K)}=\mathcal{A}^{(K)}(r), \quad \mathcal{A}_{ \pm t 1}=\mathcal{A}_{ \pm}(r), \quad \mathcal{F}_{ \pm \mu \nu \rho}=2 Q_{5 \pm} \epsilon_{\mu \nu \rho}
\end{aligned}
$$

where $\epsilon$ is the unit 3-sphere volume form. It is easy to see that the magnetic ansatz for $\mathcal{F}_{ \pm \mu \nu \rho}$ automatically solves the corresponding equation of motion and that the functions $\mathcal{A}^{(K)}(r)$ and $\mathcal{A}_{ \pm}(r)$ satisfy

$$
\begin{aligned}
\frac{d \mathcal{A}^{(K)}}{d r} & =2 Q_{K} e^{a+b-3 c-\frac{8}{3} \lambda} \\
\frac{d \mathcal{A}_{ \pm}}{d r} & =2 Q_{1 \pm}\left(f_{ \pm}(T)\right)^{-1} e^{a+b-3 c+\frac{4}{3} \lambda-4 \nu}
\end{aligned}
$$

The field equations for the remaining fields can be derived from an effective one-dimensional action $S=S\left[\phi_{6}, \lambda, \nu, T, a, b, c\right]$. Performing the change of variable $d \tau=-2 e^{-3 c-a+b} d r$ and the field redefinitions 30

$$
\rho=2 c+a, \quad \alpha=a-\frac{4}{3} \lambda, \quad \beta=a+\frac{2}{3} \lambda+2 \nu, \quad \gamma=a+\frac{2}{3} \lambda-2 \nu,
$$

this effective action takes the simple form

$$
S=\int d \tau\left[\frac{3}{2} \dot{\rho}^{2}-\frac{1}{2} \dot{\alpha}^{2}-\frac{1}{2} \dot{\beta}^{2}-\frac{1}{2} \dot{\gamma}^{2}-{\dot{\phi_{6}}}^{2}-\frac{1}{4} \dot{T}^{2}-V\left(\rho, \alpha, \beta, \gamma, \phi_{6}, T\right)\right]
$$


where denotes derivative with respect to $\tau$ and

$$
V=\frac{m^{2}}{16} T^{2} e^{3 \rho+\phi_{6}-(\beta+\gamma) / 2}-\frac{3}{2} e^{2 \rho}+\frac{1}{2} Q_{K}^{2} e^{2 \alpha}+h_{1}(T) e^{2 \gamma}+h_{5}(T) e^{2 \beta}
$$

with

$$
\begin{aligned}
& h_{1}(T)=\frac{1}{2}\left(Q_{1+}^{2}\left(f_{+}(T)\right)^{-1}+Q_{1-}^{2}\left(f_{-}(T)\right)^{-1}\right), \\
& h_{5}(T)=\frac{1}{2}\left(Q_{5+}^{2} f_{+}(T)+Q_{5-}^{2} f_{-}(T)\right) .
\end{aligned}
$$

The first term in the potential $V$ comes from the tachyon mass, the second term from the curvature of $S^{3}$ and the remaining terms from the Kałuża-Klein, D1-branes and D5-branes charges. The equations of motion following from the action (3.8) should be supplemented with the zero energy condition [30]

$$
\frac{3}{2} \dot{\rho}^{2}-\frac{1}{2} \dot{\alpha}^{2}-\frac{1}{2} \dot{\beta}^{2}-\frac{1}{2} \dot{\gamma}^{2}-\dot{\phi}_{6}{ }^{2}-\frac{1}{4} \dot{T}^{2}+V\left(\rho, \alpha, \beta, \gamma, \phi_{6}, T\right)=0 .
$$

To find the solution to the above system first we consider the tachyon field. For small $T$, we have $f_{+}(T) \sim 1 / f_{-}(T)$ and therefore the function $h_{1}(T)$ has an extremum at $T=T_{0}$ such that $f_{+}\left(T_{0}\right)=Q_{1+} / Q_{1-}[18]$. Similarly, the function $h_{5}(T)$ has an extremum at $T=T_{0}$ such that $f_{+}\left(T_{0}\right)=Q_{5-} / Q_{5+}$. For $Q_{p+}=Q_{p-} \equiv Q_{p}(p=1$ or 5$)$ we have $f_{+}\left(T_{0}\right)=1$ which is solved by $T_{0}=0$ and it is consistent with the assumption that $T$ is small (more generally if $f_{+}=f_{-}^{-1}$ and $f_{+}(0)=1$ the argument will follow, e.g. for $f_{ \pm}=e^{ \pm T}$ ). Thus, the tachyon equation is solved by $T=0$. The solution for the dilaton field $\phi_{6}$ becomes $\phi_{6}=0$ (recall that $\phi_{6}$ has its zero mode subtracted). Noting that $h_{p}(0)=Q_{p}^{2}$, the solution is similar to the D5-D1 solution of the type IIB theory obtained in [30 using the above method. In terms of the ten-dimensional theory fields we have

$$
\begin{aligned}
d s_{10}^{2}= & \left(H_{1} H_{5}\right)^{-\frac{1}{2}}\left(-d t^{2}+d x_{1}^{2}+\left(\frac{r_{0}}{r}\right)^{2}\left(\cosh (\beta) d t+\sinh (\beta) d x_{1}\right)^{2}\right) \\
& +\left(H_{1} H_{5}\right)^{\frac{1}{2}}\left(d r^{2}+r^{2} d \Omega_{3}^{2}\right)+H_{1}^{\frac{1}{2}} H_{5}^{-\frac{1}{2}} d x^{I} d x_{I}, \\
e^{2 \phi}= & \frac{H_{1}}{H_{5}}, \quad T=0, \\
\mathcal{F}_{3 \pm}= & \frac{2 Q_{1}}{r^{3}} H_{1}^{-2} d t \wedge d x_{1} \wedge d r+2 Q_{5} \epsilon,
\end{aligned}
$$

where

$$
\sqrt{2} Q_{p}=r_{p}^{2}, \quad Q_{K}=r_{0}^{2} \sinh (2 \beta) \equiv r_{K}^{2}, \quad H_{p}=1+\left(\frac{r_{p}}{r}\right)^{2}, \quad h=1-\left(\frac{r_{0}}{r}\right)^{2} .
$$


Notice that we are considering the dilute gas regime $r_{p} \gg r_{0}, r_{K}$. The fields $(\alpha, \beta, \gamma, \rho)$ corresponding to the solution (3.12) are

$$
e^{\alpha}=h^{\frac{1}{2}} H_{K}^{-1}, \quad e^{\beta}=h^{\frac{1}{2}} H_{5}^{-1}, \quad e^{\gamma}=h^{\frac{1}{2}} H_{1}^{-1}, \quad e^{\rho}=r^{2} h^{\frac{1}{2}} .
$$

Next we discuss the issue of stability of the background (3.12). We consider small perturbations of the tachyon field $T$ around $T_{0}=0$. The leading quadratic terms in the potential $V$ are seen to be

$$
V_{2}=h\left[\frac{m^{2}}{8} r^{6}\left(H_{1} H_{5}\right)^{\frac{1}{2}}+Q_{1}^{2}\left(f_{+}^{\prime}(0)\right)^{2} H_{1}^{-2}+Q_{5}^{2}\left(f_{+}^{\prime}(0)\right)^{2} H_{5}^{-2}\right] \frac{T^{2}}{2}+\mathcal{O}\left(T^{3}\right) .
$$

Asymptotically the background (3.12) is just Minkowsky space which is not stable because of the tachyon field. However, we are interested in the near-horizon geometry where we expect the tachyonic instability to be cured. The near-horizon region corresponds to the limit $r \rightarrow r_{0}$ such that the dilute gas condition $r_{p} \gg r_{0}, r_{K}, r$ holds. In this limit the metric in (3.12) becomes

$$
\begin{aligned}
d s_{10}^{2}= & \frac{r^{2}}{r_{1} r_{5}}\left(-d t^{2}+d x_{1}^{2}\right)+\frac{r_{0}^{2}}{r_{1} r_{5}}\left(\cosh (\beta) d t+\sinh (\beta) d x_{1}\right)^{2} \\
& +\frac{r_{1} r_{5}}{r^{2}-r_{0}^{2}} d r^{2}+r_{1} r_{5} d \Omega_{3}^{2}+\frac{r_{1}}{r_{5}} d x^{I} d x_{I} .
\end{aligned}
$$

This geometry is the product of the BTZ black hole which is locally $A d S_{3}$ and $S^{3} \times T^{4}$. In this background the potential $V_{2}$ becomes

$$
V_{2}=\left(r^{2}-r_{0}^{2}\right) r_{0}^{2}\left[\frac{m^{2}}{8} r_{1} r_{5}+\left(f_{+}^{\prime}(0)\right)^{2}\right] \frac{T^{2}}{2}+\mathcal{O}\left(T^{3}\right) .
$$

Thus, the tachyon is stabilized for $r_{1} r_{5} \leq 4 \alpha^{\prime}$. But this is precisely the region where the $\alpha^{\prime}$ corrections to the gravity approximation are expected to be important. However, because the $A d S_{3}$ and $S^{3}$ radii are equal the Weyl tensor for this background cancels. Assuming that the $\alpha^{\prime}$ corrections can be written in terms of the Weyl tensor we expect this geometry to be an exact solution of the theory [32]. Also, the stability condition for the tachyon field is expected to hold 18 .

Next we quantize the parameters in the gravitational solution. This can be done by relating the quanta of $\mathrm{D} 5_{ \pm}$-brane, $\mathrm{D} 1_{ \pm}$-brane and Kałuża-Klein charge with the parameters

\footnotetext{
${ }^{4}$ Note that in a $A d S$ background tachyons with sufficiently small negative mass square are allowed [31]. However, this fact does not change the above condition significantly 18 .
} 
$r_{5}, r_{1}, r_{0}$ and $\beta$. The result is

$$
\begin{aligned}
& N_{5}=N_{5 \pm}=\frac{1}{4 \pi^{2} g \alpha^{\prime}} \int_{S^{3}} \mathcal{F}_{3 \pm}=\frac{r_{5}^{2}}{\sqrt{2} g \alpha^{\prime}}, \\
& N_{1}=N_{1 \pm}=\frac{v}{4 \pi^{2} g \alpha^{\prime}} \int_{S^{3} \times T^{4}} \star \mathcal{F}_{3 \pm}=\frac{v r_{1}^{2}}{\sqrt{2} g \alpha^{\prime}}, \\
& N_{L, R}=\frac{R_{1}^{2} v}{4 g^{2} \alpha^{\prime 2}} r_{0}^{2} e^{ \pm 2 \beta},
\end{aligned}
$$

where $v=\left(R_{6} \ldots R_{9}\right) / \alpha^{\prime 2}$. Comparing with the type II case we have an extra factor of $1 / \sqrt{2}$ in the expressions for $N_{5}$ and $N_{1}$. This factor arises because the type $0 \mathrm{D} p$-brane tension $T_{p}$ is related to the type II $\mathrm{D} p$-brane tension $T_{p}^{I I}$ by $T_{p}=T_{p}^{I I} / \sqrt{2}$ and because the parameter $r_{p}$ is related to the mass of $N_{p} \mathrm{D} p_{+}$-branes and $N_{p}$ D $p_{-}$-branes (more

precisely we have $\frac{2 \pi^{2}}{\kappa_{5}^{2}} \frac{r_{p}^{2}}{2}=N_{p} \frac{T_{p}^{I I}}{\sqrt{2}} V_{p}$ ). We remark that the six-dimensional string coupling is $g_{6}=g / \sqrt{v}$ and that the condition to stabilize the tachyon derived above can be written as $g_{6} \sqrt{N_{1} N_{5}} \leq 2 \sqrt{2}$.

We are now in position to calculate the Bekenstein-Hawking entropy in terms of the quantized charges. The result is

$$
S_{B H}=\frac{4 \pi^{3}}{\kappa_{5}^{2}} r_{1} r_{5} \cosh (\beta)=2 \pi \sqrt{2 N_{1} N_{5}}\left(\sqrt{N_{L}}+\sqrt{N_{R}}\right) .
$$

Notice that there is an extra factor of $\sqrt{2}$ in comparison with the type IIB case.

\section{The $\mathrm{D} 5_{ \pm}-\mathrm{D} 1_{ \pm}$worldvolume theory}

In this section we study the worldvolume theory of the $\mathrm{D} 5_{ \pm^{-}} \mathrm{D} 1_{ \pm}$brane system. Our main goal is to derive the entropy formula (3.19) by considering a specific branch of this theory. We shall see that the field theory for the $\mathrm{D} 5_{ \pm}-\mathrm{D} 1_{ \pm}$system is a projection of the type IIB field theory for the D5-D1 brane system.

We start by analyzing the worldvolume field content on the $\mathrm{D} 5_{ \pm}-\mathrm{D} 1_{ \pm}$bound state. However, it is convenient to study instead the field theory on the T-dual D $9_{ \pm}-\mathrm{D} 5_{ \pm}$bound state. As explained in section two, strings with both ends on + charged or - charged D-branes give worldvolume bosons while strings with one end on a + charged D-brane and the other end on a - charged D-brane give worldvolume fermions. Schematically we may write

$$
\begin{array}{rll}
\text { Bosons } & \left(5_{ \pm}, 5_{ \pm}\right) ;\left(9_{ \pm}, 9_{ \pm}\right) ;\left(5_{ \pm}, 9_{ \pm}\right) ;\left(9_{ \pm}, 5_{ \pm}\right), \\
\text {Fermions }: & \left(5_{ \pm}, 5_{\mp}\right) ;\left(9_{ \pm}, 9_{\mp}\right) ;\left(5_{ \pm}, 9_{\mp}\right) ;\left(9_{ \pm}, 5_{\mp}\right)
\end{array}
$$


where a $\left(5_{+}, 5_{+}\right)$string represents a string with both ends on a D $5_{+}$-brane and so on. The corresponding massless modes that determine the field content of this gauge theory aref:

- $\left(5_{ \pm}, 5_{ \pm}\right)$strings: One gauge vector $A_{\alpha}^{(5,5)}(\alpha=0,1, \ldots, 5)$ and four scalar fields $\phi_{(5,5)}^{I}$ $(I=6, \ldots, 9)$ in the adjoint representation of $U_{+}\left(N_{1}\right) \times U_{-}\left(N_{1}\right)$.

- $\left(9_{ \pm}, 9_{ \pm}\right)$strings: One gauge vector $A_{\alpha}^{(9,9)}$ and four scalar fields $\phi_{(9,9)}^{I}$ in the adjoint representation of $U_{+}\left(N_{5}\right) \times U_{-}\left(N_{5}\right)$.

- $\left(5_{+}, 9_{+}\right)$and $\left(9_{+}, 5_{+}\right)$strings: One $S U(2)$ doublet $\chi$ describing two complex scalars in the $\left(N_{1}, \bar{N}_{5}\right)$ of $U_{+}\left(N_{1}\right) \times U_{+}\left(N_{5}\right)$.

- $\left(5_{-}, 9_{-}\right)$and $\left(9+, 5_{-}\right)$strings: One $S U(2)$ doublet $\chi^{\prime}$ describing two complex scalars in the $\left(N_{1}, \bar{N}_{5}\right)$ of $U_{-}\left(N_{1}\right) \times U_{-}\left(N_{5}\right)$.

- $\left(5_{+}, 5_{-}\right)$strings: Two Weyl spinors $\Psi_{i}^{(5,5)}(i=1,2)$ in the $\left(N_{1}, \bar{N}_{1}\right)$ of $U_{+}\left(N_{1}\right) \times U_{-}\left(N_{1}\right)$.

- $\left(5_{-}, 5_{+}\right)$strings: Two Weyl spinors $\Psi_{i}^{\prime(5,5)}$ in the $\left(\bar{N}_{1}, N_{1}\right)$ of $U_{+}\left(N_{1}\right) \times U_{-}\left(N_{1}\right)$.

- $\left(9_{+}, 9_{-}\right)$strings: Two Weyl spinors $\Psi_{i}^{(9,9)}$ in the $\left(N_{5}, \bar{N}_{5}\right)$ of $U_{+}\left(N_{5}\right) \times U_{-}\left(N_{5}\right)$.

- $\left(9_{-}, 9_{+}\right)$strings: Two Weyl spinors $\Psi_{i}^{\prime(9,9)}$ in the $\left(\bar{N}_{5}, N_{5}\right)$ of $U_{+}\left(N_{5}\right) \times U_{-}\left(N_{5}\right)$.

- $\left(5_{+}, 9_{-}\right)$and $\left(9_{-}, 5_{+}\right)$strings: One Weyl spinor $\lambda$ in the $\left(N_{1}, \bar{N}_{5}\right)$ of $U_{+}\left(N_{1}\right) \times U_{-}\left(N_{5}\right)$.

- $\left(5_{-}, 9_{+}\right)$and $\left(9_{+}, 5_{-}\right)$strings: One Weyl spinor $\lambda^{\prime}$ in the $\left(N_{1}, \bar{N}_{5}\right)$ of $U_{-}\left(N_{1}\right) \times U_{+}\left(N_{5}\right)$.

Now we show that the gauge theory for the $\mathrm{D} 9_{ \pm}-\mathrm{D} 5_{ \pm}$system can be obtained as a projection of the analogous supersymmetric gauge theory of the type IIB theory, i.e. a $N=$ $1, D=6$ supersymmetric gauge theory. We start with two vectormultiplets in the adjoint of $U\left(2 N_{1}\right)$ and $U\left(2 N_{5}\right)$ together with two hypermultiplets in the same representation of the gauge groups. These fields can be written as a vector gauge field, two Weyl spinors and four scalars, i.e. $\left(A_{\alpha}^{(5,5)}, \Psi_{i}^{(5,5)}, \phi_{(5,5)}^{I}\right)$ and $\left(A_{\alpha}^{(9,9)}, \Psi_{i}^{(9,9)}, \phi_{(9,9)}^{I}\right)$ in the adjoint of $U\left(2 N_{1}\right)$ and $U\left(2 N_{5}\right)$, respectively. This is the field content of the $N=2, D=6$ supersymmetric gauge theory that arises from the reduction on $T^{4}$ of the $N=1, D=10$ super YangMills theory. The theory has another hypermultiplet $(\chi, \lambda)$ where $\chi$ is a $S U(2)$ doublet describing two complex scalars and $\lambda$ a Weyl spinor both transforming in the $\left(2 N_{1}, \overline{2 N}_{5}\right)$ of $U\left(2 N_{1}\right) \times U\left(2 N_{5}\right)$.

\footnotetext{
${ }^{5}$ The subscripts + and - in the gauge groups allow us to distinguish between the $U(N)$ factors corresponding to + or - charged branes.
} 
The $\mathrm{D} 9_{ \pm}-\mathrm{D} 5_{ \pm}$field theory is obtained by a $Z_{2}$ projection of the above theory. We take the $Z_{2}$ action to be $(-1)^{F} \mathcal{I}$ where $F$ is the fermionic number and $\mathcal{I}$ is the conjugation by $\operatorname{Diag}(I,-I)$ where $I$ is the $N_{1} \times N_{1}$ or the $N_{5} \times N_{5}$ identity matrix [18]. This projection breaks the gauge symmetry $U\left(2 N_{1}\right)$ to $U_{+}\left(N_{1}\right) \times U_{-}\left(N_{1}\right)$ and $U\left(2 N_{5}\right)$ to $U_{+}\left(N_{5}\right) \times U_{-}\left(N_{5}\right)$. The action of this projection on the fields $\left(A_{\alpha}^{(n, n)}, \Psi_{i}^{(n, n)}, \phi_{(n, n)}^{I}\right)(n=5,9)$ gives the fields $\left(A_{\alpha}^{(n, n)}, \Psi_{i}^{(n, n)}, \Psi_{i}^{\prime(n, n)}, \phi_{(n, n)}^{I}\right)$ of our theory and it is similar to the D $3_{ \pm}$-brane case discussed in [18]. To determine the action on the hypermultiplet $(\chi, \lambda)$ we decompose these fields in $N_{1} \times N_{5}$ blocks. The conjugation by $\mathcal{I}$

$$
\left(\begin{array}{ll}
A & B \\
C & D
\end{array}\right) \rightarrow\left(\begin{array}{cc}
I_{N_{1}} & 0 \\
0 & -I_{N_{1}}
\end{array}\right)\left(\begin{array}{cc}
A & B \\
C & D
\end{array}\right)\left(\begin{array}{cc}
I_{N_{5}} & 0 \\
0 & -I_{N_{5}}
\end{array}\right)=\left(\begin{array}{cc}
A & -B \\
-C & D
\end{array}\right),
$$

together with the $(-1)^{F}$ action leaves us with the fields $\chi, \chi^{\prime}$ and $\lambda, \lambda^{\prime}$ previously discussed.

To calculate the entropy associated with the gravitational background (3.16) we assume that the dynamics of this black hole is determined by the strings stretching between $\mathrm{D} 1_{ \pm^{-}}$ and $\mathrm{D} 5 \pm^{-}$-branes. This is analogous to what happens in the type IIB case. Thus, the system is described by $2 \times\left(4 N_{1} N_{5}\right)$ massless bosons arising from the fields $\chi$ and $\chi^{\prime}$ and by $2 \times\left(4 N_{1} N_{5}\right)$ massless fermions arising from the fields $\lambda$ and $\lambda^{\prime}$, where we are considering the reduction of our gauge theory to two dimensions. Hence, we have a conformal field theory with central charge $c=2 \times(4+2) N_{1} N_{5}$. This effective theory has to be conformal because of the $A d S_{3}$ character of the dual type 0B background. If we excite this system with $N_{L, R}$ units of left- and right-moving momentum, we have the usual asymptotic formula for the entropy

$$
S=\pi \sqrt{c \frac{2}{3}}\left(\sqrt{N_{L}}+\sqrt{N_{R}}\right)=2 \pi \sqrt{2 N_{1} N_{5}}\left(\sqrt{N_{L}}+\sqrt{N_{R}}\right),
$$

which is valid for $N_{L, R} \gg N_{1} N_{5}$ and exactly reproduces the result (3.19). The previous condition may be relaxed by considering winding D-branes [33].

Finally, we justify the assumption that the black hole dynamics is determined by open strings stretching between $\mathrm{D} 1_{ \pm^{-}}$and $\mathrm{D} 5_{ \pm^{-}}$-branes. We assume that the dual field theory is the projection $(-1)^{F} \mathcal{I}$ of the dual field theory in the type II case, i.e. the Higgs branch of the $N=1, D=6$ supersymmetric gauge theory (compactified on $T^{4}$ ) discussed above. This branch corresponds to exciting the hypermultiplets while the vectormultiplets are in their vacuum state. To analyze the projected theory we consider for simplicity only the bosonic degrees of freedom. The D-terms of the original supersymmetric theory become in 
the projected theory

$$
\begin{aligned}
& D_{I J}^{a}=f_{b c}^{a}\left(\phi_{I}^{b} \phi_{J}^{c}+\frac{1}{2} \epsilon_{I J K L} \phi_{K}^{b} \phi_{L}^{c}\right)+\chi^{\dagger} T^{a} \Gamma_{I J} \chi, \\
& D_{I J}^{a^{\prime}}=f_{b^{\prime} c^{\prime}}^{a^{\prime}}\left(\phi_{I}^{b^{\prime}} \phi_{J}^{c^{\prime}}+\frac{1}{2} \epsilon_{I J K L} \phi_{K}^{b^{\prime}} \phi_{L}^{c^{\prime}}\right)+\chi^{\prime \dagger} T^{a^{\prime}} \Gamma_{I J} \chi^{\prime},
\end{aligned}
$$

where we are using the notation in 20]. The indices $a, b, c$ run over the gauge group generators of $U_{+}\left(N_{1}\right)$ and $U_{+}\left(N_{5}\right)$ and the indices $a^{\prime}, b^{\prime}, c^{\prime}$ of $U_{-}\left(N_{1}\right)$ and $U_{-}\left(N_{5}\right)$. To define a vacuum of this theory we must set the potential

$$
V=\sum_{a I J}\left(D_{I J}^{a}\right)^{2}+\sum_{a^{\prime} I J}\left(D_{I J}^{a^{\prime}}\right)^{2}
$$

to zero. This gives $2 \times\left(3 N_{1}^{2}\right)+2 \times\left(3 N_{5}^{2}\right)$ conditions. The number of independent gauge transformations is $2 N_{1}^{2}+2 N_{5}^{2}$. Since the number of massless bosons in the projected Higgs branch is $2 \times\left(4 N_{1} N_{5}+4 N_{1}^{2}+4 N_{5}^{2}\right)$ we are left with $2 \times\left(4 N_{1} N_{5}\right)$ bosons parameterizing the moduli space of vacua. But these are precisely the number of bosons associated with the open strings stretching between $\mathrm{D} 1_{ \pm^{-}}$and $\mathrm{D} 5_{ \pm^{-}}$-branes.

It is interesting to relate the above description to the instanton moduli space approximation of [26]. In the latter description we view the $\mathrm{D} 1_{ \pm}$-branes as instantons in the D5 $\pm_{-}$-branes gauge theory. This theory is the $U_{+}\left(N_{5}\right) \times U_{-}\left(N_{5}\right)$ six-dimensional gauge theory with four adjoint scalars and four bifundamental Weyl spinors (two in the $\left(N_{5}, \bar{N}_{5}\right)$ and two in the $\left.\left(\bar{N}_{5}, N_{5}\right)\right)$. The $N_{1} \mathrm{D} 1_{ \pm}$-branes are seen as instantons in this gauge theory with instanton number $\nu=N_{1+}+N_{1-}=2 N_{1}$. Our results predict that the moduli space of these instantons is given by a conformal field theory with $2 \times\left(4 N_{1} N_{5}\right)$ species of bosons and $2 \times\left(4 N_{1} N_{5}\right)$ species of fermions, i.e. with central charge $c=12 N_{1} N_{5}$.

\section{Conclusion}

Let us start by summarizing the results presented in this paper. We started by considering intersecting D-branes in type 0 string theory. We calculated the cylinder amplitudes for intersecting $\mathrm{D} p$ - and $\mathrm{D} p^{\prime}$-branes with the result that open strings terminating on + (or -) charged D-branes give worldvolume bosons while open strings terminating on a + charged D-brane and on a - charged D-brane give worldvolume fermions. We concluded that the intersecting rules for the $\mathrm{D} p_{ \pm}$-brane bound states are identical to the intersecting rules for the D-branes of the type II theories. In the remainder of this paper we analyzed in detail the $\mathrm{D} 5_{ \pm}-\mathrm{D} 1_{ \pm}$brane system of the type 0B theory. We showed that the near-horizon geometry for this D-brane bound state is the $A d S_{3} \times S^{3} \times T^{4}$ space (the $A d S_{3}$ factor may 
be replaced by the BTZ black hole) which was argued to be a stable and exact solution of the type $0 \mathrm{~B}$ gravitational equations. We proceeded by describing the worldvolume field theory on this D-brane system. It turned out that this gauge theory is a $Z_{2}$ projection of the analogous theory for the D5-D1 brane system of the type IIB theory. By projecting the Higgs branch of the latter theory we correctly reproduced the Bekenstein-Hawking entropy formula calculated in the gravitational analysis. Note that this agreement between the gravitational and microscopic entropy calculations may be seen to arise because the type 0B theory is a orbifold of the type IIB theory. As a consequence, the gravitational solution is similar to the type IIB case but with the doubling of the $\mathrm{R}-\mathrm{R}$ fields, while the gauge theory is obtained by a $Z_{2}$ projection of the type IIB D5-D1 gauge theory.

Finally, we note that because the rules for intersecting the $\mathrm{D} p_{ \pm}$-brane bound states are the same as for the type II theories, there are many results that are expected to hold in the type 0 theories. In particular, we expect to have $D=4$ black holes corresponding to other intersecting $\mathrm{D} p_{ \pm}$-branes, e.g. four intersecting D $3_{ \pm}$-branes [34. Also, we expect to have rotating black holes [35]. Furthermore, we expect that more generally the $\mathrm{D} p_{ \pm}$-branes can intersect at $S U(N)$ angles [36]. The corresponding gravitational background can be derived by using the results in refs. [37, 38, 39]. Also, there should be bound states of branes with constant worldvolume magnetic fields and corresponding black hole solutions 40 .

\section{Acknowledgments}

I would like to thank Michael Gutperle and Lori Paniak for discussions and specially Igor Klebanov for very useful discussions and for reading a draft of the paper. This work was supported by FCT (Portugal) under programme PRAXIS XXI and by the NSF grant PHY-9802484. 


\section{References}

[1] L. Dixon and J. Harvey, Nucl. Phys. B274 (1986) 93.

[2] N. Seiberg and E. Witten, Nucl. Phys. B276 (1986) 272.

[3] J. Polchinski, String Theory, Vol. 2, Cambridge University Press, 1998.

[4] I.R. Klebanov and A.A. Tseytlin, D-Branes and Dual Gauge Theories in Type 0 Strings, hep-th/9811035.

[5] A.M. Polyakov, The Wall of the Cave, hep-th/9809057.

[6] J.M. Maldacena, Adv. Theor. Math. Phys. 2 (1998) 231.

[7] S.S. Gubser, I.R. Klebanov and A.M. Polyakov, Phys. Lett. B428 (1998) 105.

[8] E. Witten, Adv. Theor. Math. Phys. 2 (1998) 253.

[9] J. Minahan, Glueball Mass Spectra and Other Issues for Supergravity Duals of QCD Models, hep-th/9811156.

[10] I.R. Klebanov and A.A. Tseytlin, Asymptotic Freedom and Infrared Behavior in the Type 0 String Approach to Gauge Theory, hep-th/9812089.

[11] M.R. Garousi, String Scattering from D-branes in Type 0 Theories, hep-th/9901085.

[12] K. Zarembo, Coleman-Weinberg Mechanism and Interaction of D3-Branes in Type 0 String Theory, hep-th/9901106.

[13] J. Minahan, Asymptotic Freedom and Confinement from Type 0 String Theory, hepth/9902074.

[14] I.I. Kogan and G. Luzón, Scale Invariance of Dirac Condition $g_{e} g_{m}=1$ in Type 0 String Approach to Gauge Theory, hep-th/9902086.

[15] G. Ferretti and D. Martelli, On the construction of gauge theories from non critical type 0 strings, hep-th/9811208.

[16] E. Alvarez and C. Gomez, Non-Critical Confining Strings and the Renormalization Group, hep-th/9902012. 
[17] A. Armoni, E. Fuchs and J. Sonnenschein, Confinement in $4 D$ Yang-Mills Theories from Non-Critical Type 0 String Theory, hep-th/9903090.

[18] I.R. Klebanov and A.A. Tseytlin, A Non-supersymmetric Large N CFT from Type 0 String Theory, hep-th/9901101.

[19] A.A. Tseytlin and K. Zarembo, Effective potential in non-supersymmetric $S U(N) \times$ $S U(N)$ gauge theory and interactions of type 0 D3-branes, hep-th/9902095.

[20] J.M. Maldacena, Black Holes in String Theory, Ph.D. Thesis, hep-th/9607235.

[21] O. Bergman and M.R. Gaberdiel, Nucl. Phys. B499 (1997) 183.

[22] M. Bianchi and A. Sagnotti, Phys. Lett. B247 (1990) 517.

[23] A. Sagnotti, Some Properties of Open - String Theories, hep-th/9509080; Nucl. Phys. Proc. Suppl. B56 (1997) 332.

[24] C. Angelantonj, Phys. Lett. B444 (1998) 309.

[25] G. Lifschytz, Phys. Lett. B388 (1996) 720.

[26] A. Strominger and C. Vafa, Phys. Lett. B379 (1996) 99.

[27] C. Callan and J.M. Maldacena, Nucl. Phys. B472 (1996) 591.

[28] M. Billó, B. Craps and F. Roose, On D-branes in Type 0 String Theory, hepth/9902196.

[29] M. Green, J. Harvey and G. Moore, Class. Quant. Grav. 14 (1997) 47.

[30] C.G. Callan, S.S. Gubser, I.R. Klebanov and A.A. Tseytlin, Nucl. Phys. B489 (1997) 65.

[31] P. Breitenlohner and D.Z. Freedman, Ann. Phys. 144 (1982) 249.

[32] S.S. Gubser, I.R. Klebanov and A.A. Tseytlin, Nucl.Phys. B534 (1998) 202.

[33] J.M. Maldacena and L. Susskind, Nucl. Phys. B475 (1996) 679.

[34] I.R. Klebanov and A.A. Tseytlin, Nucl.Phys. B475 (1996) 179.

[35] M. Cvetič and F. Larsen, Nucl. Phys. B531 (1998) 239; Microstates of FourDimensional Rotating Black Holes from Near-Horizon Geometry, hep-th/9805146. 
[36] M. Berkooz, M.R. Douglas and R.G. Leigh, Nucl. Phys. B480 (1996) 265.

[37] J.C. Breckenridge, G. Michaud and R.C. Myers, Phys. Rev. D56 (1997) 5172.

[38] V. Balasubramanian, F. Larsen, R.G. Leigh, Phys. Rev. D57 (1998) 3509.

[39] M.S. Costa and M. Cvetič, Phys. Rev. D56 (1997) 4834-4843.

[40] M.S. Costa and M.J. Perry, Nucl. Phys. B520 (1998) 205; Nucl. Phys. B524 (1998) 333; M.S. Costa, J. High Energy Phys. 11 (1998) 007. 